\title{
Multicolored ZnO Nanowire Architectures on Trenched Silicon Substrates
}

\author{
Pu-Xian Gao, ${ }^{\dagger, *}$ J. L. Lee, ${ }^{\S}$ and Zhong L. Wang*,† \\ School of Materials Science and Engineering, Georgia Institute of Technology, Atlanta, Georgia 30332-0245, \\ Sandia National Laboratories, Livermore, California 94551, and Institute of Materials Science \& Department \\ of Chemical, Materials and Biomolecular Engineering, University of Connecticut, Storrs, Connecticut \\ 06269-3136
}

Received: June 8, 2007; In Final Form: July 20, 2007

\begin{abstract}
Well-tailored three-dimensional (3D) $\mathrm{ZnO}$ nanowire architectures have been successfully grown on $\mathrm{Si}$ microtrenches fabricated using nanoimprinting lithography by a low-temperature hydrothermal approach. Au nanoparticles or $\mathrm{ZnO}$ nanofilms were used as templates to tailor the orientation ordered nanowire growth normal to the microtrench surface. Au produced sparse nanowire growth, while $\mathrm{ZnO}$ seeds created densely packed growth. Optically, other than displaying a primary color when viewed from one incident angle, the 3D nanowire architecture periodically displayed multiple primary color domains covering all microtrenches and the local orientation ordered nanowire arrays. A pre-growth annealing of $\mathrm{ZnO}$ nanoseeds resulted in nonuniformity and non-periodic distribution of the grown nanoarchitectures and thus reduced the multicolor effect.
\end{abstract}

Photonic crystals comprised of regularly spaced nanobuilding blocks such as nanoparticles (NPs), nanowires (NWs), and nanobeams (NBs) can offer unique optical properties and therefore have promising applications in nanolasers, ${ }^{1,2}$ nanophotonics, and quantum computing. A number of techniques have been used for the fabrication of three-dimensional (3D) photonic crystals including silicon micromachining, ${ }^{3}$ holographic lithography, ${ }^{4}$ self-assembly, ${ }^{5,6}$ glancing-angle deposition, ${ }^{7,8}$ autocloning, ${ }^{9}$ and biotemplate replication. ${ }^{10-13}$ However, for these techniques, major drawbacks include the postprocessed polycrystalline structure, high processing cost, and low defect controllability. ${ }^{14}$ It is a challenge to fabricate single crystalline 3D nanophotonic crystals at low-cost, large scale, and high efficiency.

Here in this work, by combining the nanoimprinting lithography (NIL) technique and low-temperature chemical synthesis approach, a new strategy has been developed for fabricating large scale 3D ordered NW architectures. On the basis of $\mathrm{Si}$ microtrenches with a two-dimensional (2D) cross-sectional profile, we successfully tailored the growth of 3D $\mathrm{ZnO} \mathrm{NW}$ architectures using low-temperature hydrothermal synthesis. Optically, 3D NW architectures exhibited unique multicolored nanodiffraction effects distinct from the 2D NW arrays on flat $\mathrm{Si}$ surface. This work demonstrates that in combination with this low-temperature synthesis technique, well-defined 3D substrates produced using NIL can be used to engineer welloriented 3D NW architectures as potential multicolored nanodisplays, nanophotonic interconnects, and nanowaveguides.

The growth of $\mathrm{ZnO}$ NWs was conducted by suspending the $\mathrm{Au}$ or $\mathrm{ZnO}$ seeds modified substrates in a Pyrex glass bottle filled with an equal molar aqueous solution of zinc nitrate hexahydrate $\left(\mathrm{Zn}\left(\mathrm{NO}_{3}\right)_{2} \cdot 6 \mathrm{H}_{2} \mathrm{O}, 0.01 \mathrm{M}\right)$ and hexamethylenetetramine $\left(\mathrm{C}_{6} \mathrm{H}_{12} \mathrm{~N}_{4}, 0.01 \mathrm{M}\right)$ at $80{ }^{\circ} \mathrm{C} .{ }^{15}$ The reaction time was

\footnotetext{
* Corresponding author. E-mail: zhong.wang@mse.gatech.edu.

Georgia Institute of Technology.

IMS/CMBE, University of Connecticut.

$\S$ Sandia National Laboratories.
}

(a)
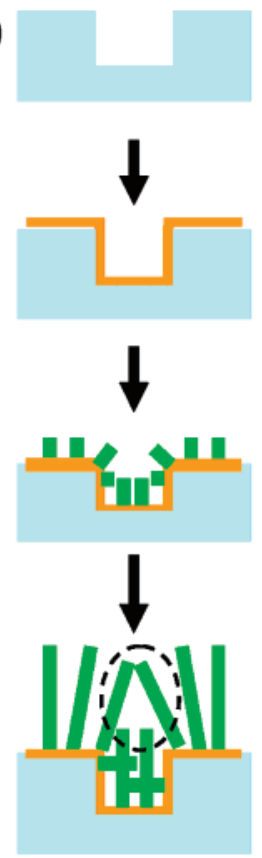

$\mathrm{Si}$
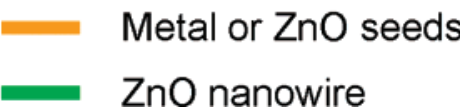

Figure 1. Schematic diagram illustrating the fabrication process for 3D NW architectures, based on (a) rectangular-shaped and (b) triangular-shaped microtrench arrays as defined by NIL.

2-18 h for both substrates coated with $\mathrm{Au}$ or layers with $\mathrm{ZnO}$ seeds. After reaction, the substrates were removed from the solution, rinsed with deionized water, and dried in air at $80{ }^{\circ} \mathrm{C}$ overnight. The structure and morphology of $\mathrm{ZnO}$ NWs were characterized by scanning electron microscopy (SEM, LEO 1530 and $1550 \mathrm{FEG}$ at 5 and $10 \mathrm{kV}$ ) and transmission electron 

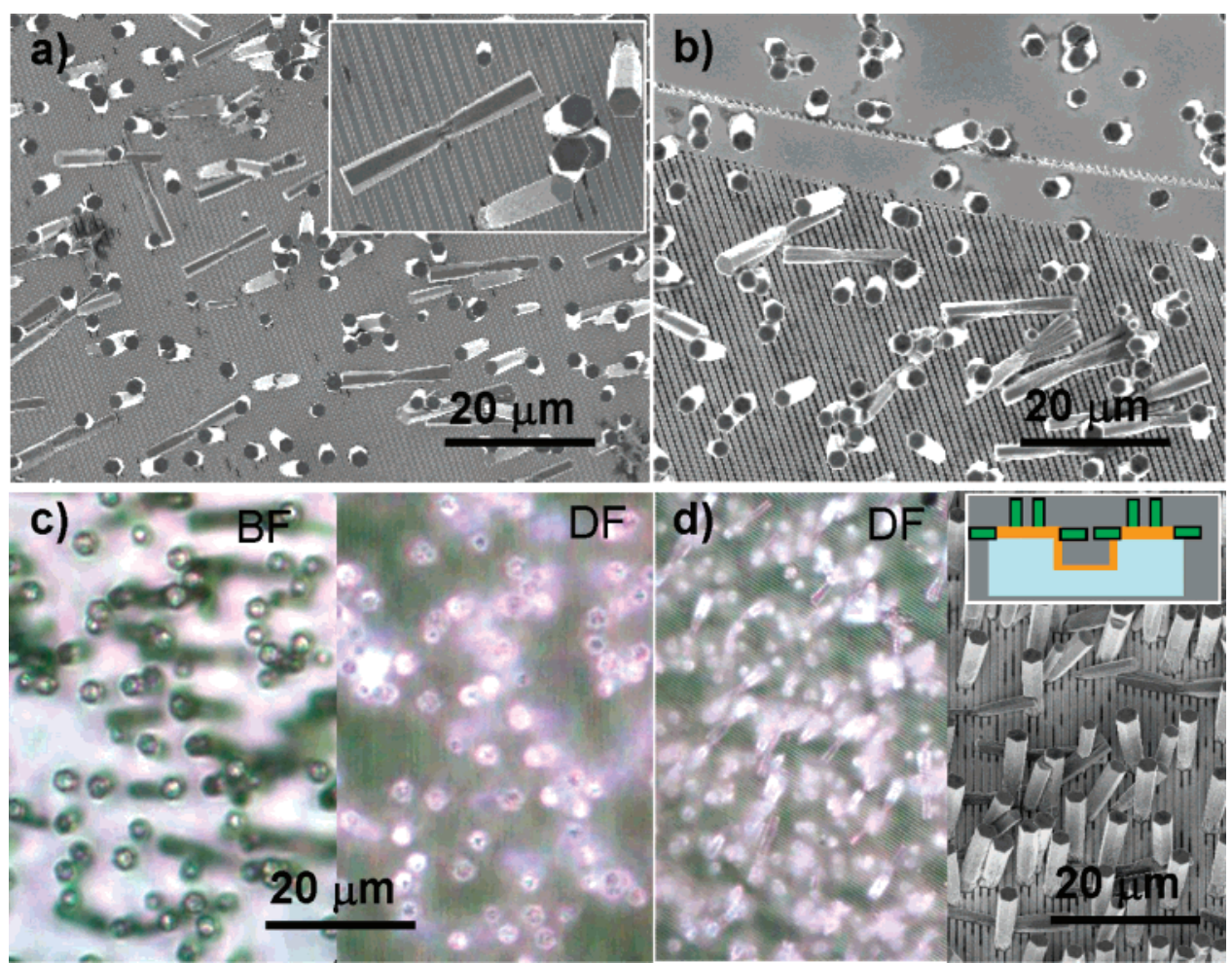

Figure 2. SEM images $(\mathrm{a}, \mathrm{b})$ and optical micrographs $(\mathrm{c}, \mathrm{d})$ displaying the typical morphology of NWs grown on a rectangular-shaped trenched Si substrate coated with Au.

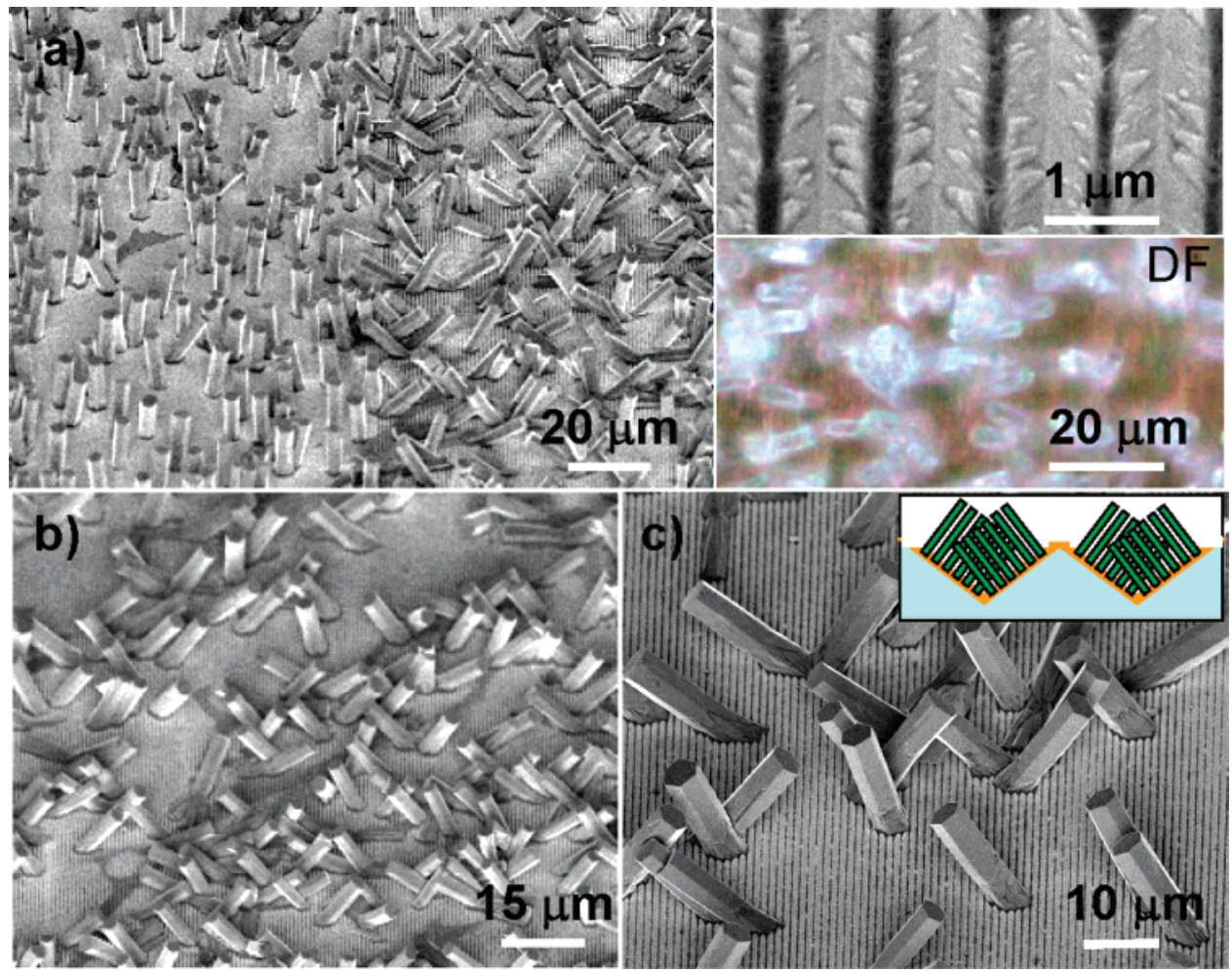

Figure 3. Set of SEM images and optical micrographs displaying the typical morphology of NWs grown on a triangular-shape trenched Si substrate coated with Au.

microscopy (TEM, Hitachi HF-2000 at $200 \mathrm{kV}$ ). A Nikon Eclipse L150 optical microscope attached with a charge-coupled device (CCD) camera together with an image processing computer was used for optical imaging at magnifications of $100 \times, 200 \times, 500 \times$, and $1000 \times$. Reflection spectroscopy was conducted on a Beckman DU640 UV-vis-NIR spectrophotometer with wavelength coverage of $200-1100 \mathrm{~nm}$.
Figure 1 is a schematic diagram illustrating the fabrication process of 3D NW architectures. Two microtrench geometries defined by NIL (Nanonex NX-2000) have been used. One is a rectangular-shaped trench (Figure 1a), and the other is a triangular-shaped trench (Figure 1b). Both microtrench arrays have the same periodicity of $\sim 1 \mathrm{um}$. Before NW growth, $\mathrm{Si}$ substrates containing these two types of microtrenches had a 


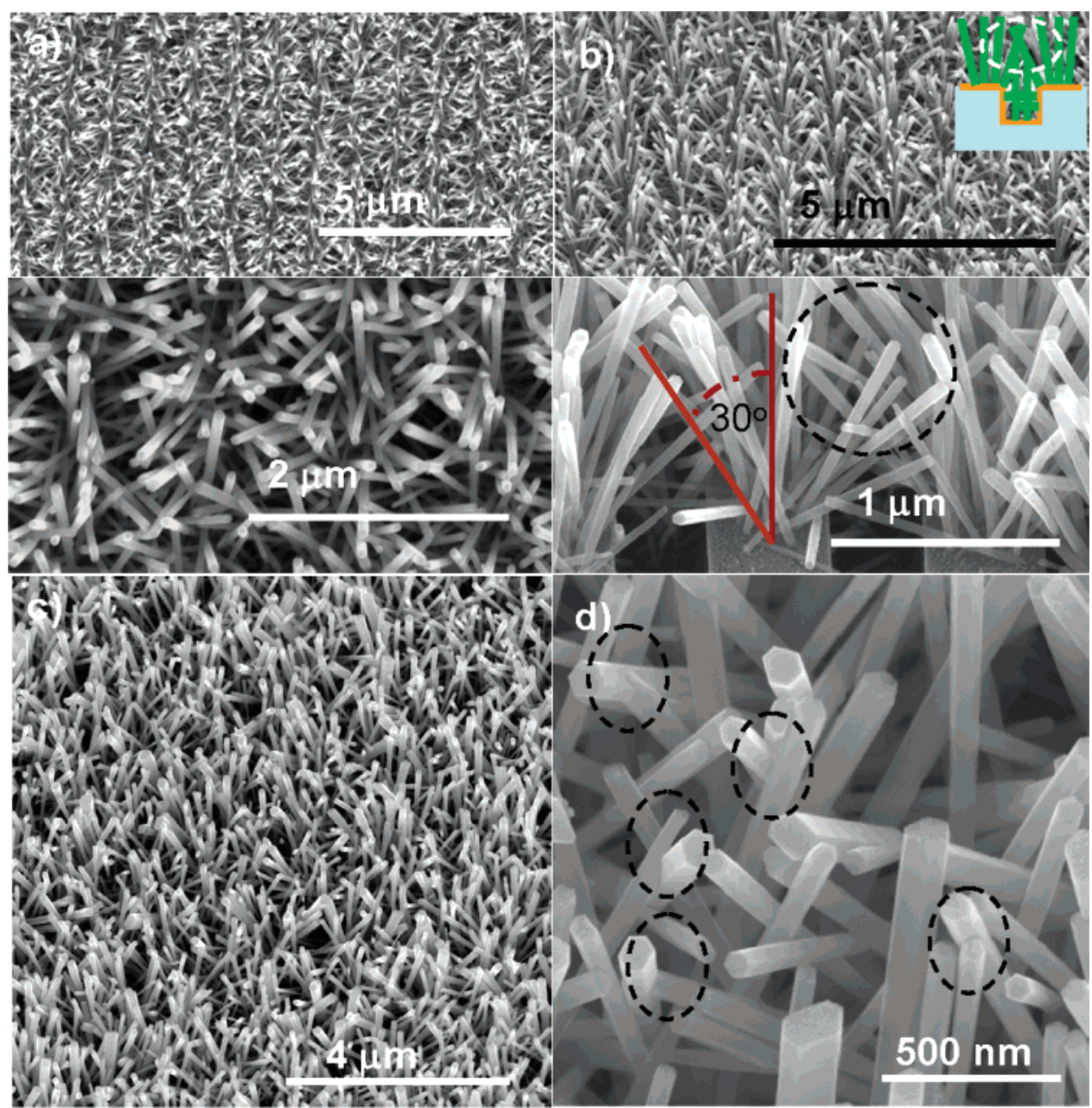

Figure 4. A set of SEM images of as-grown NWs on rectangular-shape trenches coated with $50 \mathrm{~nm}$ of $\mathrm{ZnO}$ seeds without (a,b) and with (c,d) postannealing.

$50 \mathrm{~nm}$ thick layer of $\mathrm{Au}$ or $\mathrm{ZnO}$ seeds deposited on them by magnetron sputtering. As the growth proceeded, $\mathrm{ZnO}$ NWs were controlled by the template of either the Au layer or the $\mathrm{ZnO}$ seed layer to promote growth normal to the local substrate surface. ${ }^{15}$ In this way, 3D NW architecture can be achieved by virtue of alternating two orientations normal to each other (Figure 1a) or with certain angles (Figure 1b) depending on the $2 \mathrm{D}$ cross-sectional profile of the microtrenches. But because of the space limitation for the NWs, when NWs grow beyond a certain density, the NWs will tend to compromise and grow vertically, instead of conforming to growth normal to the substrate surface (inclined growth is also considered in Figure 1a). Furthermore, it is also expected that intersection phenomena will happen as the growth continues, as indicated in the dotted elliptical regions.

A plan view SEM image in Figure 2a illustrates the typical morphology of NWs grown on a rectangular-shaped microtrench Si substrate coated with Au. Clearly, the NWs grew sparsely but not in a periodic way and were tailored by the microtrench geometry to grow along one of of two primary orientations. One orientation is horizontal and parallel to the microtrench bottom surface and normal to the trenchlength, as indicated in the inset picture. The other is vertical and parallel to the sidewalls of microtrenches and normal to the trench length. The hexagonal top surfaces of vertical NWs suggested the growth direction of $\mathrm{ZnO} \mathrm{NWs}$ is along the $c$ axis of wurtzite $\mathrm{ZnO}$. Each individual horizontal NW generally has a uniform dumbbell structure, as indicated by red arrowheads. Another plane view
SEM image in Figure $2 b$ displayed the NW growth around the boundary between the flat Si surface and the microtrenched surface of the substrates. On the flat Si surface (top part of Figure 2b), the growth gave rise to a 2D vertical array of NWs. Because of the existence of rectangular-shaped microtrenchs, a 3D NW architecture formed by alternation of two orientations conforming to the $2 \mathrm{D}$ rectangle cross-sectional profile is seen in the bottom part of Figure $2 b$. Figure $2 c$ are typical optical micrographs in dark field (DF, left) and bright field (BF, right) at $500 \times$ that are focused on the top surfaces of the vertical NW array. A quite uniform shadow of the horizontal NW array was clearly seen in the form of dark-green lines, while the vertical NW array was recorded as dark-blue circle patterns with the incident light beam normal to the top surfaces. The bright field optical micrograph instead revealed a bright dotted pattern for vertical NW arrays, which is due to the large angle reflection from their mirrored top hexagonal surfaces. In Figure 2d, a set of side-view optical (left) and SEM (right) images clearly revealed a 3D architecture comprised by the vertical NW arrays and horizontal NW arrays, as illustrated in the inset.

On the triangular-shaped microtrench $\mathrm{Si}$ substrate coated with $\mathrm{Au}$, the growth of NWs also conformed to the cross-sectional profile of the NIL-defined microtrench patterns. Figure $3 \mathrm{a}$ is a side view of the grown 3D NW architectures. On the left, a vertically aligned NW array was grown on the flat Si surface; on the right side, a 3D NW architecture is presented with $\mathrm{ZnO}$ NWs growing along two alternate directions defined by the triangle cross-sectional profile. A top-view optical micrograph 

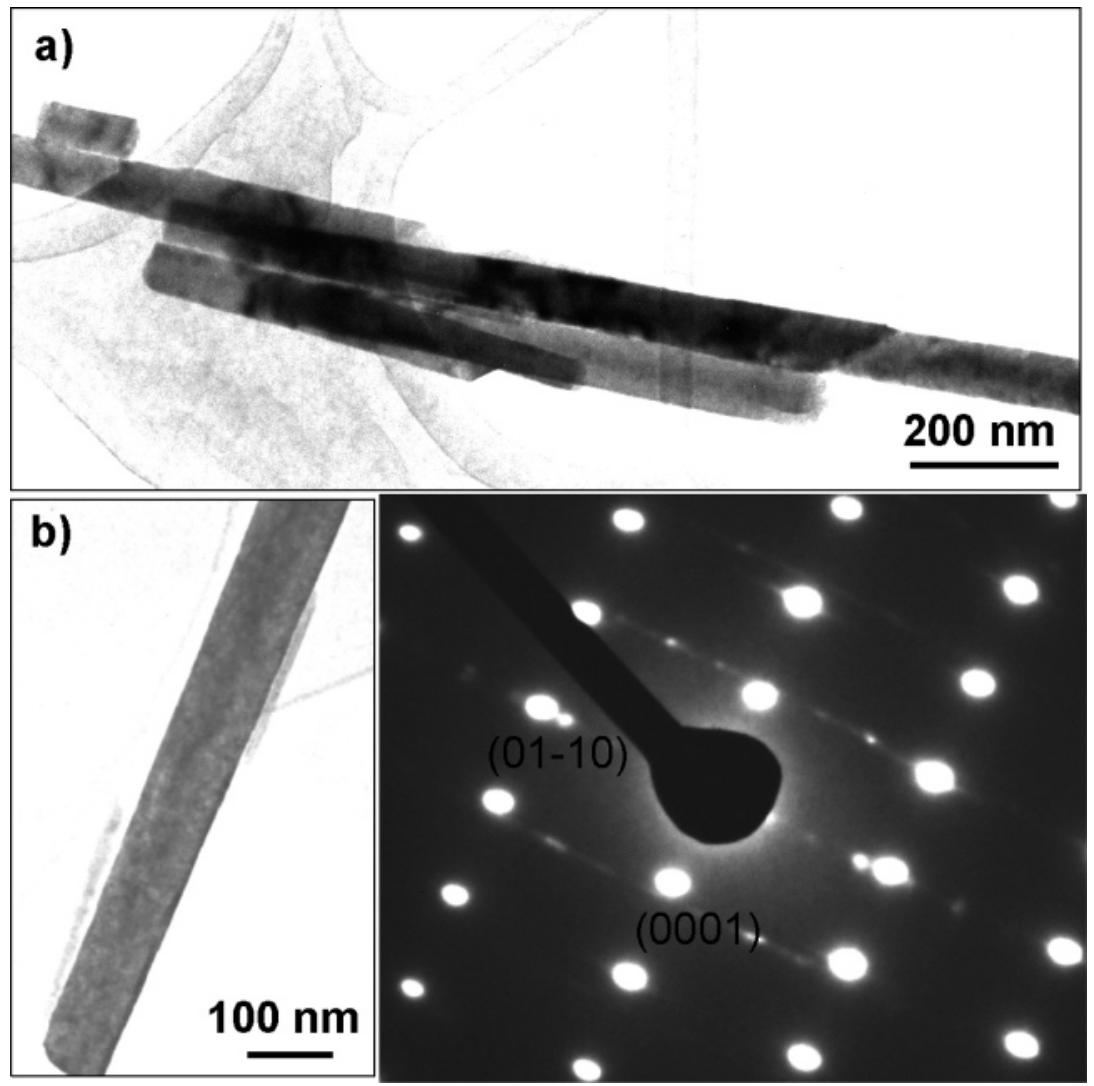

Figure 5. (a) Typical low magnification TEM images and (b) electron diffraction pattern corresponding to an individual ZnO NW.

in the bottom right inset of Figure 3a was blurred because of the tilted side walls and hence inclined NWs with respect to the incident light beam. The magnified tilted view SEM images shown in Figure 3b,c were a clearer portrait of the 3D NW architectures with defined alternating orientations but without a notable periodicity due to the sparse distribution of NWs. Despite a size distribution of NWs in the range of $1.5-3 \mu \mathrm{m}$ (Figure 3c) that is larger than the periodicity of the microtrench of $\sim 1 \mu \mathrm{m}$ (top right inset of Figure $3 \mathrm{a}$ ), the triangle microtrench profile still defines the two alternating growth orientations of the NW array.

To inherit the periodicity of the microtrenches, a layer of sputtered $\mathrm{ZnO}$ seeds was chosen instead of an $\mathrm{Au}$ coating as an alternate template for $\mathrm{ZnO} \mathrm{NW}$ growth. The results turned out to be very successful. When the Au layer was replaced with a layer of $\mathrm{ZnO}$ seeds, the 3D NW architectures grew much denser under the same growth conditions, and the NWs are much thinner. Figure $4 a, b$ is respectively top-view and side-view SEM images of the as-grown NWs on rectangular-shaped microtrenches coated with $50 \mathrm{~nm}$ of $\mathrm{ZnO}$ seeds without postanneal processing. Clearly, the periodicity of microtrench array has been inherited after the growth, where the 3D NW array exhibited the same periodicity of $\sim 1 \mu \mathrm{m}$. The intersection phenomena become frequent as NW lengths are of a uniform $\sim 2 \mu \mathrm{m}$, twice the periodicity. The NWs have a diameter of $\sim 80 \mathrm{~nm}$. The circled region in Figure $4 \mathrm{a}, \mathrm{b}$ represents some areas with significant intersection of NWs with different orientations. The tilted view in Figure $4 \mathrm{~b}$ indicated a very good periodicity with the 3D NWs architecture from the top surface to the bottom of the microtrenches. The densely packed NWs have an inclined orientation distribution ranging from $0^{\circ}$ to $90^{\circ}$ with respect to the normal direction of the top surface of the substrate, while the majority of the NW orientations are $0-30^{\circ}$, which might be due to the fact that the trenched areas are relatively localized and their geometry might limit access of solution reactants to establish NW growth locations. Figure 4c,d is respectively topview and side-view SEM images of NWs grown on rectangularshaped microtrench substrate coated with $\mathrm{ZnO}$ seeds and annealed. Despite a similar size distribution as the NWs grown using unannealed $\mathrm{ZnO}$ seeds, this tailored $\mathrm{NW}$ architecture has to some extent lost the periodicity of the underlying $\mathrm{Si}$ microtrenches. The lost preferred crystal orientations for $\mathrm{ZnO}$ seeds after annealing might contribute to the loss of periodicity of NW architectures. A more random orientation distribution of seeds might have led to more frequent intersection events, as indicated by the dotted circle regions. Interconnecting junction regions have formed in these areas when two or more NWs met each other.

To find the crystallinity and structure of the as-grown NWs, TEM characterization was conducted. Figure 5a is a typical low magnification TEM image. The diameter of each NW is $\sim 80$ $\mathrm{nm}$, and the length is $\sim 1.5-3 \mu \mathrm{m}$. Each NW was a single crystal with very good crystallinity and grew along [0001] enclosed by $\{01 \overline{10}\}$ faceted side surfaces, as revealed by the electron diffraction pattern shown in Figure 5b.

From the above structure and morphology characterization results, there is no doubt that 3D NW architectures can inherit the underlying 3D microtrench surface profiles by controlling the growth directions of NWs using $\mathrm{Au}$ or $\mathrm{ZnO}$ seed layers. When the density and NW diameter were controlled to fit into the dimensions of the periodic microtrench arrays, the periodicity of microtrench pattern can be inherited as well.

Optically, periodicity and orientation modulation are two keys for realizing specific photonic functions such as light trapping and guiding. ${ }^{16}$ To find out if the inherited periodicity and orientation alternation from microtrench substrates have any optical characteristics in these tailored 3D $\mathrm{ZnO} \mathrm{NW}$ architectures, angular reflection measurements were performed on three 

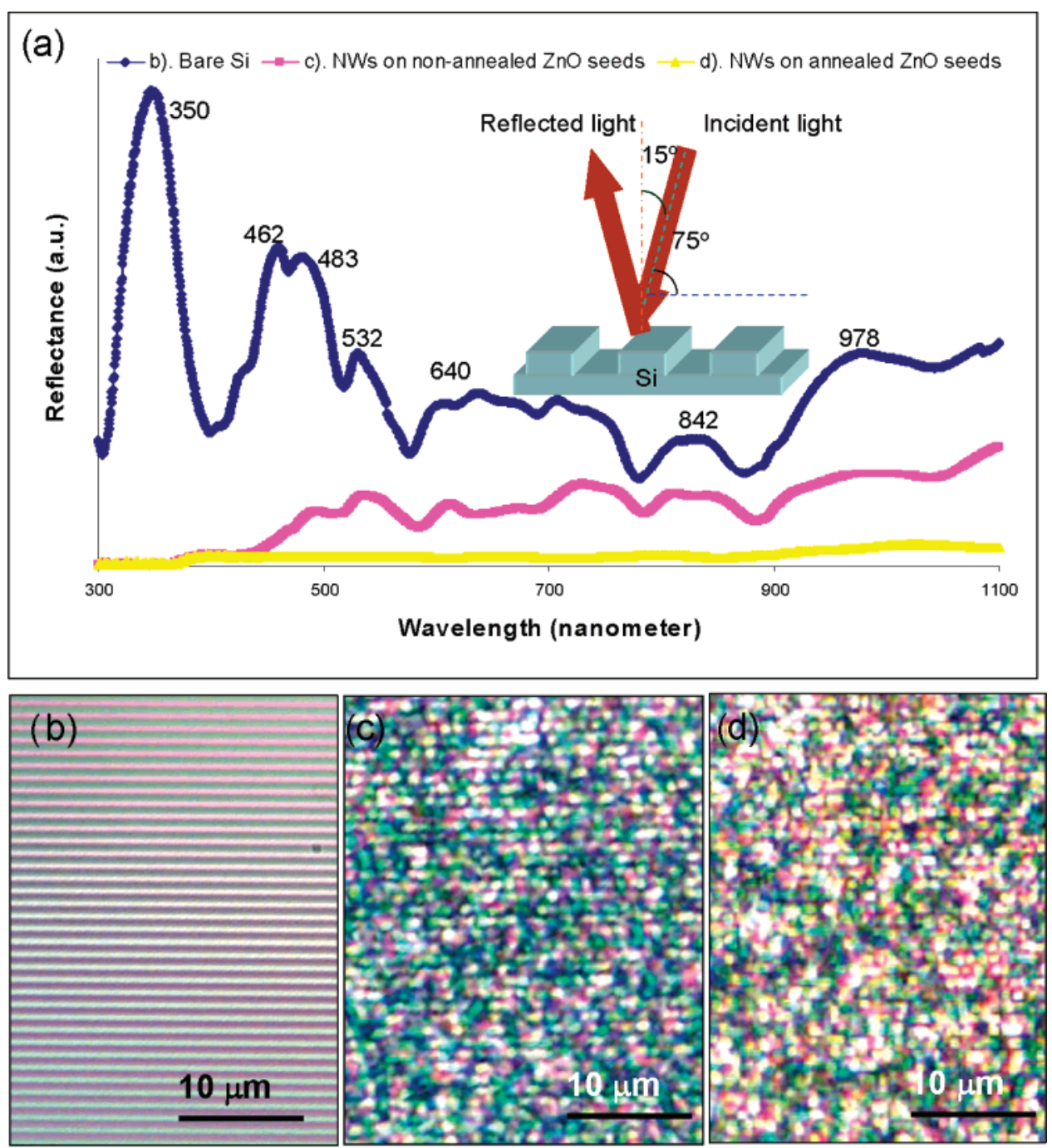

Figure 6. (a) Typical collected reflectance spectra from three types of rectangular-shaped trench samples under an incident light beam at a $15^{\circ}$ angle from the normal direction of the substrate surface. $(\mathrm{b}-\mathrm{d})$ Optical micrographs corresponding to the bare Si substrate, 3D ZnO NW architectures on seeds coated substrate without annealing, and seeds coated substrate with annealing, respectively.

samples for comparison. The three samples were respectively bare $\mathrm{Si}$ with rectangular-shaped microtrenches, 3D ZnO NWs on a rectangular-shaped microtrenched substrate coated with unannealed $\mathrm{ZnO}$ seeds, and $3 \mathrm{D} \mathrm{ZnO}$ NWs on a rectangularshaped microtrenched substrate that was coated with $\mathrm{ZnO}$ seeds and annealed at $400{ }^{\circ} \mathrm{C}$. The experiments were conducted in a wavelength range from UV $(300 \mathrm{~nm})$ to visible $(400-800 \mathrm{~nm})$ and near-infrared $(800-1100 \mathrm{~nm})$ to reveal a full range of light interaction with the 3D NW architectures, $\mathrm{Si}$, and relevant nearby air cavities. To achieve maximum interaction between the incident light and the substrates, the reflectance was measured at $15^{\circ}$ with respect to the normal direction of the flat Si substrate surface, with the incident light beam normal to the trench lines, as illustrated in the inset of Figure 6a. Therefore, the incident light was propagating along a major NW axis direction and strongly interacting with the periodic 3D NW architecture.

Figure $6 \mathrm{a}$ is the collected reflectance spectra. For comparison purposes, the reflectance spectrum of the bare silicon substrate is presented as a dark blue line, which revealed large reflections in the whole experimental wavelength range. This explains why the background of the optical image in Figure $6 \mathrm{~b}$ appeared white. The relatively stronger reflection happened in the light blue (462 and $483 \mathrm{~nm})$ and UV (350 $\mathrm{nm})$ range, leading to slightly tinted cyan and violet colors on the white background. As a compari- son, the ZnO NW samples appear to have a strong ability to absorb the UV range wavelength including $350 \mathrm{~nm}$, as evidenced by UV range spectra $(300-390 \mathrm{~nm})$ with negligible reflectance in Figure 6a. In the infrared wavelength region, despite some absorption, the reflectance peaks and valleys repeated for the $\mathrm{ZnO} \mathrm{NWs}$ on the unannealed Si substrate. It is suggested that at infrared wavelengths, the light still can propagate along the surface of $\mathrm{Si}$ substrate with a $\sim 2 \mu \mathrm{m}$ thick top layer of $\mathrm{ZnO}$ NWs, through the transparent $\mathrm{ZnO}$ NWs single crystals without too much absorption and diffusive scattering loss. Compared with the unannealed sample, the annealed sample has shown much more significant absorption over the full wavelength range. The reason for this phenomenon might be due to the more random orientation of the NWs (Figure 4c,d) compared with the unannealed sample (Figure 4a,b). As a result, the randomly oriented $\mathrm{ZnO} \mathrm{NW}$ increase the extent of diffusive scattering and absorption, therefore reducing the overall reflectance dramatically.

There is a dramatic color difference between the two $\mathrm{ZnO}$ NW samples when they are observed with the naked eye. For $\mathrm{ZnO} \mathrm{NWs}$ grown on unannealed $\mathrm{ZnO}$ seed/Si substrates, the sample looks colorful when viewed from different angles, while the sample with an annealed $\mathrm{ZnO}$ seed/Si substrate appeared white without any other specific colors when viewed from different angles. Undoubtedly, the diffusive scattering over the 

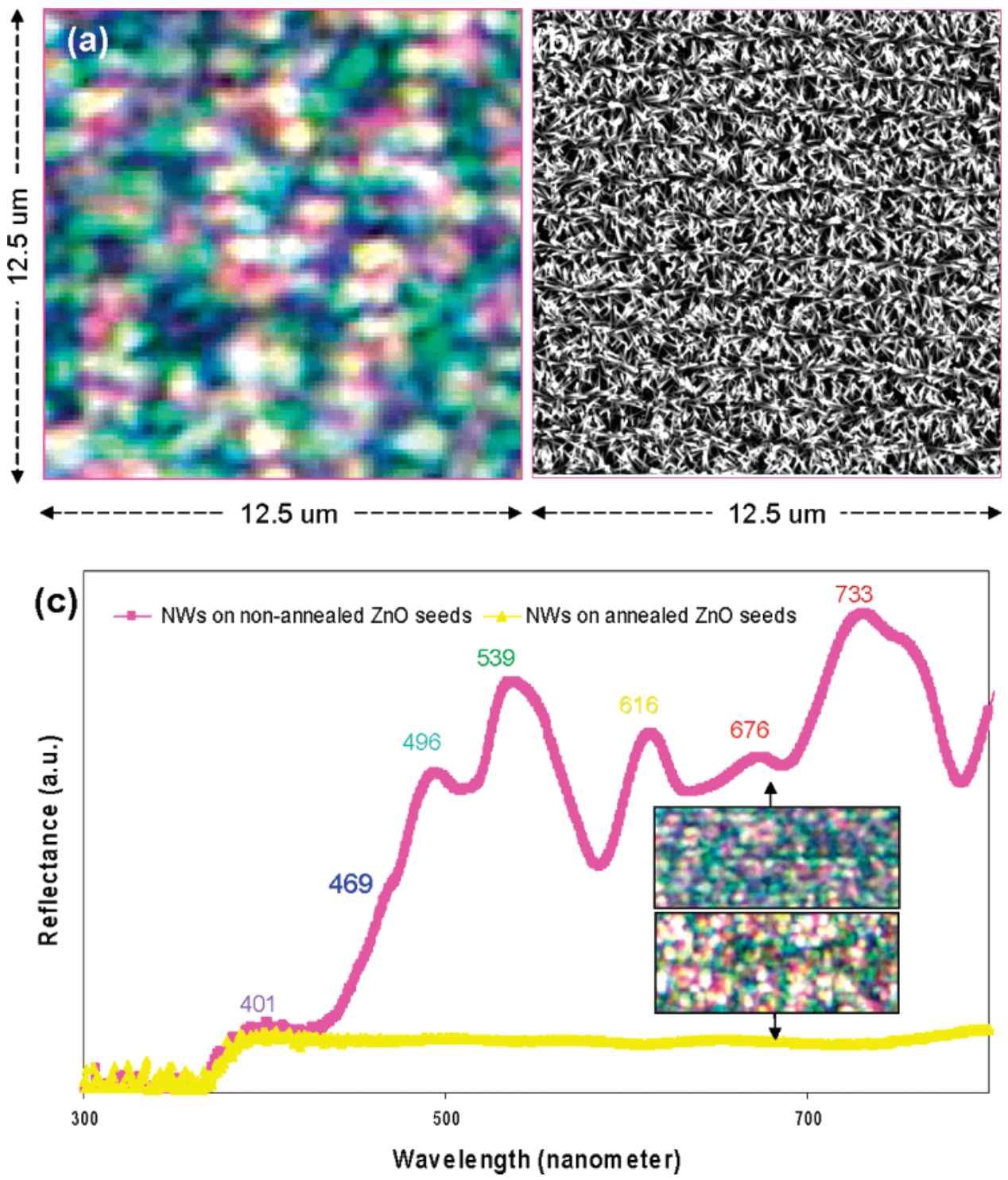

Figure 7. (a,b) Optical and an electron micrograph corresponding to an area of $12.5 \mu \mathrm{m} \times 12.5 \mu \mathrm{m}$ from the NW architecture grown on seeds coated substrates without annealing. (c) Comparison of reflectance spectrum in visible wavelength range between nonannealed sample and annealed sample.

whole wavelength range is likely due to the random orientations of the NWs despite the existence of the local orientation ordering (Figure 6d, optical image at $1000 \times$ ). Under the reflectance optical microscope, different local reflectance wavelengths can be resolved. Figure $6 \mathrm{c}, \mathrm{d}$ is two optical micrographs corresponding respectively to the unannealed and annealed samples. The two local regions exhibited different color distributions. The one with more ordered 3D NWs archtectures (Figure 6c) clearly has a more uniform and colorful reflectance including red, orange, green, cyan, blue, and violet, while for the randomly oriented 3D NWs architectures, the diffusive scattering has led to fewer distinct color displays with red, yellow, blue, and violet, and more unresolved white regions.

To reveal the correlation of color domains with the tailored 3D NW architectures, more detailed optical characterization has been conducted. We carefully compared optical micrographs in correlation with the electron micrographs covering the same size area of $\mathrm{ZnO} \mathrm{NW}$ architectures. Figure $7 \mathrm{a}, \mathrm{b}$ is a pair of optical and electron micrographs corresponding to an area of $12.5 \mu \mathrm{m} \times 12.5 \mu \mathrm{m}$ on the unannealed sample. As limited by the resolution of the optical microscope used in this study, the reflectance effect cannot be recorded in a region smaller than $\sim 100 \mathrm{~nm}$. That is to say that each color domain was emitted by a bunch (tens) of NWs with a certain orientation. The resolved color domains in Figure 7 a turned out to be able to cover all of the primary colors including red, orange, yellow, green, cyan, blue, and violet in the visible wavelength range $(380-740 \mathrm{~nm})$. As shown by the labeled peaks in Figure $7 \mathrm{c}$ for the unannealed sample, the reflectance peaks covered all of the primary colors except yellow. Each domain color is a little bit diffuse instead of completely uniform, suggesting a certain narrow orientation distribution for the NWs comprising one color domain. This is consistent with the SEM imaging results in Figure 4a,b, where the orientations of the NWs ranged from $0^{\circ}$ to $90^{\circ}$ to the direction normal to the substrate top surface, with the majority of the NWs oriented around $15^{\circ}$ (Figure $4 \mathrm{~b}$ ). Overall, the color domain shape is not very uniform in Figure $7 \mathrm{a}$, but it still preserved the periodicity of $1 \mu \mathrm{m}$ like the tailored 3D NW architecture in Figure 7b. Although the domain shape can be circular, square, or rectangular, the domain width is generally confined to the range of $300-500 \mathrm{~nm}$. Since the two strongest reflectance peaks for the unannealed sample are 733 and $539 \mathrm{~nm}$ (Figure 7c), which are respectively in the red range $(625-740 \mathrm{~nm})$ and green range $(500-565 \mathrm{~nm})$, red and green 
color domains have been the major color domains for Figure 7a. Compared with the unannealed sample, the annealed sample exhibited a very low reflectance spectrum, with one relatively significant peak at $401 \mathrm{~nm}$, in the violet wavelength range. As discussed previously, much more frequent diffusive scattering and absorption of full range spectrum on the sample have led to a white appearance at a larger scale. As shown in the inset bottom optical micrograph, the background domain colors on a microscopic scale were red, yellow, green, violet, and white.

From the above discussions, a layer of ordered $3 \mathrm{D} \mathrm{ZnO} \mathrm{NW}$ architectures have engineered the white $\mathrm{Si}$ substrate under reflected light into a periodic multicolored map comprised of periodic color domains. It is suggested that the periodicity of color domains and color uniformity can be precisely engineered by tuning the periodicity of 3D substrates as well as the orientation distribution of NW growth. It is envisioned that through more detailed design and processing of the 3D surface profiles of the substrates, as well as more directed design and synthesis of NW architectures with desired dimensions, separations, and orientations, specifically sized, localized, shaped, and colored nanodomains can be achieved.

In summary, well-tailored 3D $\mathrm{ZnO} \mathrm{NW}$ architectures have been successfully fabricated on the basis of the NIL defined Si microtrenches using low-temperature hydrothermal synthesis. During the synthesis, Au or $\mathrm{ZnO}$ nanofilms have been used as the templates to tailor the orientationally ordered growth of NWs normal to the local trench surface profile. The transparent semiconductor NW architectures grew sparsely upon using Au film as the templates, as compared with densely packed ones on $\mathrm{ZnO}$ seeds, and each NW was a single crystal along [0001] and enclosed by $\{01 \overline{1} 0\}$ facet surfaces. Optically, 3D ZnO NW architectures exhibit unique nanodiffraction effects from the 2D NW arrays on flat Si surface. Other than displaying a primary color when viewed from a specific incident angle, the 3D NW architecture can display periodic, multiple primary color domains covering the entire visible wavelength range by combining microtrenches with local orientationally ordered NW arrays. A pre-growth anneal of $\mathrm{ZnO}$ nanoseeds resulted in nonuniformity and nonperiodic distribution of the tailored nanoarchitectures and thus reduced the multicolor effect.

Acknowledgment. The authors thank A. A. Talin for producing the nanoimprinted $\mathrm{Si}$ substrates. This work was supported in part by Sandia National Laboratories, a multiprogram laboratory operated by Sandia Corporation, a Lockheed Martin Company, for the U.S. Department of Energy's National Nuclear Security Administration under Contract No. DE-AC0494AL85000.

\section{References and Notes} 143.

(1) Joannopoulos, J. D.; Villeneuve, P. R.; Fan, S. Nature 1997, 386,

(2) Huang, M. H.; Mao, S.; Feick, H.; Yan, H. Q.; Wu, Y. Y.; Kind, H.; Weber, E.; Russo, R.; Yang, P. D. Science 2001, 292, 1897.

(3) Fleming, J. G.; Lin, S. Y. Opt. Lett. 1999, 24, 49.

(4) Campbell, M.; Sharp, D. N.; Harrison, M. T.; Denning, R. G.; Turberfield, A. J. Nature 2000, 404, 53.

(5) Vlasov, Y. A.; Bo, X. Z.; Sturm, J. C.; Norris, D. J. Nature 2001, $414,289$.

(6) Ozin, G. A.; Yang, S. M. Adv. Funct. Mater. 2001, 11, 95. 2,59 .

(7) Kennedy, S. R.; Brett, M. J.; Toader, O.; John, S. Nano. Lett. 2002,

(8) Zhang, G. G.; Zhao, Y. P. J. Appl. Phys. 2004, 95, 267.

(9) Kuramochi, E.; Notomi, M.; Kawashima, T.; Takahashi, J.; Takahashi, C.; Tamamura, T; Kawakami, S. Opt. Quant. Elec. 2002, 34, 53.

(10) Davis, S. A.; Burkett, S. L.; Mendelson, N. H.; Mann, S. Nature 1997, 385, 420 .

(11) Dong, A. G.; Wang, Y. J.; Tang, Y.; Ren, N.; Zhang, Y. H.; Yue, J. H.; Gao, Z. Adv. Mater. 2002, 14, 926.

(12) Cook, G.; Timms, P. L.; Spickermann, C. G. Angew. Chem., Int Ed. 2003, 42, 557.

(13) Huang, J. Y.; Wang, X. D.; Wang, Z. L. Nano. Lett. 2006, 6, 2325.

(14) Qi, M. H.; Lidorikis, E.; Rakich, P. T.; Johnson, S. G.; Joannopoulos, J. D.; Ippen, E. P.; Smith, H. I. Nature 2004, 429, 538.

(15) Gao, P. X.; Song, J. H.; Liu, J.; Wang, Z. L. Adv. Mater. 2007, 19, 67.

(16) Lonĉar, M.; Doll, T.; Vučković, J.; Scherer, A. J. Lightwave Tech. 2000, 18, 1402 . 